PHOTOMETRIC AND H-ALPHA VARIABILITY IN SOME Be STARS

S. Catalano, G. Umana

Istituto di Astronomia, Universita di Catania

Viale A. Doria, 95125 Catania

C.N.R., Gruppo Nazionale di Astronomia UdR Catania, Italy

\title{
Introduction
}

Short-term variations, typically with small amplitude $(\Delta \mathrm{m}<0.1 \mathrm{mag}$.$) , time-scales of hours or near one day and in many$ cases correlated with the rotation period have been found to be commonplace among Be stars (see Harmanec and Pavlovski 1983 for review and papers). Radial and non-radial pulsation modes have been proposed to explain this variability. However, the light curves are often doublepeaked, at first suggesting the stars may have large organized dipole magnetic fields and spots not unlike those in the Ap or Bp stars (Harmanec 1983). These rotationa1ly modulated variations are not stable, and seem to vary in both amplitude and period in the sense that intervals with well defined light curves alternate with intervals when variations are absent. This phenomenon is very reminiscent of formation and apparent relative movement in longitude of spots (groups) in some RS CVn binaries (Catalano 1983, Rodono 1986), but on a much more rapid time scale. Here we present a preliminary report of photometric and spectrographic observations of Be stars carried out at the Catania Observatory with the aim of studying short-term variations which could eventually be interpreted in terms of surface inhomogeneities.

\section{Observations and Results}

$\underline{\kappa} \underline{\text { Dra }}=\underline{H D} 109387(B 5$ IV). This star is reported as a suspected variable in the General Catalog of Variable Stars but no systematic photometric observations have been done. We observed $\kappa$ Dra in the UBV photometric system with a $30 \mathrm{~cm}$ telescope for 15 nights in April-May 1984 using HD 112429 and HD 109551 as comparison and check stars. No clear trend of variation is seen in our observations even if a significant scatter up to $0.05 \mathrm{mag}$ both in $U$ and $B-V$ from night to night is apparent. No short term variations are found during single nights in excess of the expected accuracy of the measurements, i.e. \pm $0.01 \mathrm{mag}$.

28 Cyg $=$ HD 191610 (B3.5 IV). We observed 28 Cyg on 7 nights in the period July-October 1985 at the $91 \mathrm{~cm}$ telescope using $u, v, b, y$ Stromgren filters. HD 193369 ( $36 \mathrm{Cyg}$ ) and HD 194335 were adopted as comparison and check stars, respectively. Changes from night to night 
and an average brightening of $0.05 \mathrm{mag}$ in the $\mathrm{y}$ filter associated with a reddening in b-y are consistent with an increased emission in the envelope. Data on single nights show systematic increasing or decreasing trends of $0.02-0.3 \mathrm{mag}$. Periodic analysis with discrete Fourier method (Deeming, 1975) shows that data can be phased with a period around $0.587 \mathrm{~d}$ and amplitude of $0.03 \mathrm{mag}$. This possible period agrees more with the time-scale of variability estimated by Percy et al. (1981) than with $0.7 d$ periodicity found by Spear et a1. (1981).

$\mathrm{V} 66 \mathrm{Oph}=\mathrm{HD} 164284(\mathrm{~B} 2 \mathrm{Vn})$. Cousins (1952) reported small irregular brightness fluctuations of $V 66$ Oph with amplitudes up to 0.07 mag, while two flares of 1 and 1.8 mag were detected in August 1969 by Page and Page (1970) with a time-scale of 2-3 min. We observed V66 Oph on 12 nights at the $91 \mathrm{~cm}$ telescope using $\mathrm{u}, \mathrm{v}, \mathrm{b}, \mathrm{y}$ filters. HD 161868 and HD 164353 were adopted as comparison and check stars. A steady decrease in the $y$ light and a concurrent bluer b-y is apparent. This is consistent with a fading of the envelope emission. No short-term variability is seen during single nights nor could any periodicity be detected.

Spectroscopy. Photographic spectra of $\kappa$ Dra around $H \alpha$ and $H \beta$ were obtained at a dispersion of $40 \AA / \mathrm{mm}$ with the echelle spectrograph attached to the $91 \mathrm{~cm}$ telescope in the period January-February 1984. A large change in the $\mathrm{Ha}$ emission equivalent width was seen to take place between January 26 and February 2. The emission equivalent width was $9.2 \AA$, i.e. at the level of previous measurements (Andrillat \& Fehrenbach 1982) on January 26 , reached a maximum of $38.5 \AA$ on February 1 , and faded down to $20 \AA$ on February 2. HB shows the same trend but with a smaller amplitude. The time-scale for these large changes in the level of emission is similar to that observed in the spectrum of $\mathrm{Mu}$ Cen (Peters 1986) to change from absorption to emission. However, the time-scale to change from absorption to emission spectrum in $k$ Dra seems to be about 30 years (Hubert 1971).

Acknowledgements. This work has partially been supported by the Ministero Pubblica Istruzione through the Catania University. The Osservatorio Astrofisico di Catania and The CNR - Gruppo Nazionale di Astronomia Contract n. 85.00244.02. We wish to thank C. Trigilio for the support given in the time-series analysis.

\section{References}

Andrillat, Y. \& Fehrenbach, Ch. (1982). Astron. Astrophys. Suppl., Ser., $48,93$.

Catalano, S. (1983) in Activity in Red Dwarf Stars, IAU Co11. 71 , eds.

P. B. Byrne, M. Rodono, p. 343 Reidel, Dordrecht.

Cousins, A. W. J. (1952). M.N.A.S. So. Africa 11, 9.

Page, A. A. \& Page, B. (1970). Proc. ASA 1, $32 \overline{4 .}$ 


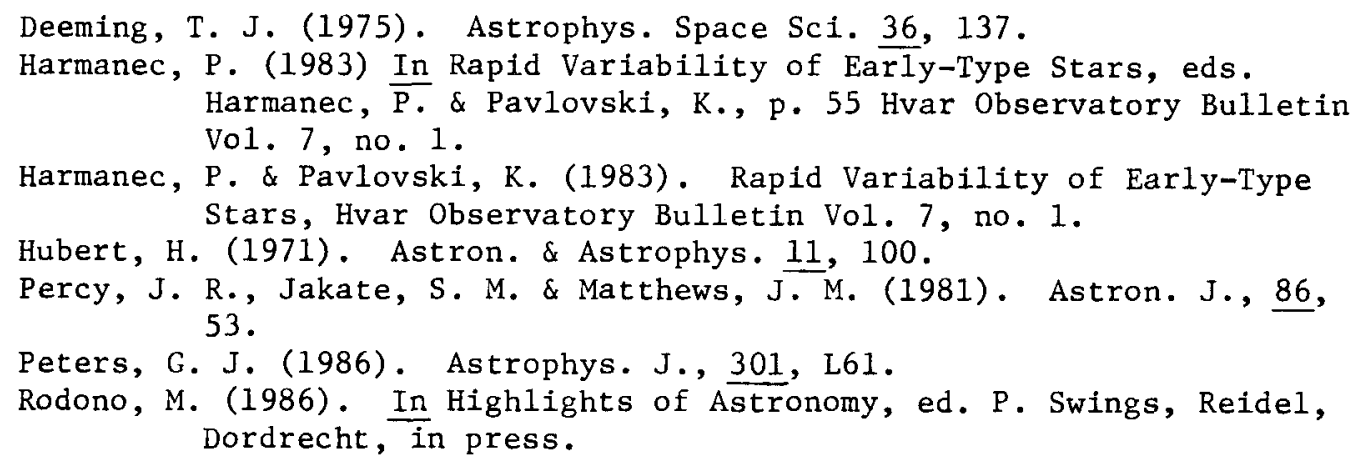

\section{DISCUSSION FOLLOWING CATALANO}

Percy:

We have two summers $(1985,1986)$ of $\mathrm{BV}$ photometric data on 28 Cyg from the University of Toronto. We have attempted to analyze the short-term variability by using three weeks of overlapping data from Toronto, Hvar, and the Automatic Photometric Telescope in Arizona. We find that the short-term variations are large (up to $0.1 \mathrm{~m}$ ), but that the power spectrum is very complex - there is no simple, single period. The analysis of this and other short-term variables (prime example: CX Dra) is greatly complicated by medium- and long-term variations.

Catalano:

Actually the power in our periodic analysis is not very high and we do not give high confidence to the $0.582 \mathrm{~d}$ period. 